\title{
Comparative Evaluation of Enamel Micro-cracks Observed before Bonding and after Debonding of Various Types of Ceramic Brackets: A SEM Study
}

\author{
${ }^{1}$ Astha Namdhari, ${ }^{2}$ VK Ravindranath, ${ }^{3}$ Vivek J Patni, ${ }^{4}$ Girish R Karandikar, ${ }^{5}$ Amol Mhatre, ${ }^{6}$ Divij Joshi, ${ }^{7}$ Prateek Daga,
}

${ }^{8}$ Aarti Madhaswar

\begin{abstract}
Introduction: Enamel integrity gets affected by the presence of micro-cracks in it and they can further create problems like stains and the accumulation of plaque on the fractured surfaces. Avoiding such iatrogenic damage to the enamel surface has been a constant challenge even with the use of metal brackets. Creating a fracture line in the base of the bracket leads to the formation of a 'weak zone'. This allows the bracket to collapse in a mesiodistal direction when debonding forces are applied rather than shattering the bracket into tiny multiple pieces. Thus, removal of the bracket having such a 'weak zone' created by making a groove in it before bracket removal arguably leaves the major amount of resin on the tooth and therefore causes less stress on enamel. This study plans to evaluate the expected beneficial effect of 'scoring' the base of the ceramic bracket before bracket removal.
\end{abstract}

Aim: To evaluate and compare the occurrence of micro-cracks in enamel observed before bonding and after debonding of various types of ceramic brackets.

Results: The difference between scored monocrystalline ceramic brackets and unscored monocrystalline ceramic brackets of both the $\mathrm{AO}$ and Ormco groups is not significant statistically $(p=0.096)$. There is a significant difference in scoring of ARI and enamel micro-cracks development. The difference of length and width between the groups (A and $B$ ) is statistically insignificant.

Conclusion: Post debonding, there was no difference significantly in the length or width of enamel micro-cracks between AO monocrystalline ceramic brackets (group A) and Ormco monocrystalline ceramic brackets (group B).

Keywords: Adhesive remnant index, Ceramic brackets, Debonding, Enamel damage.

How to cite this article: Namdhari A, Ravindranath VK, Patni VJ, Karandikar GR, Mhatre A, Joshi D, Daga P, Madhaswar A. Comparative Evaluation of Enamel Micro-cracks Observed before Bonding and after Debonding of Various

\footnotetext{
${ }^{1}$ Postgraduate Student, ${ }^{2-4}$ Professor, ${ }^{5}$ Reader, ${ }^{6-8}$ Senior Lecturer ${ }^{1}$ Department of Orthodontics, MGM Dental College and Hospital, Gwalior, Madhya Pradesh, India

2,3,5-8 Department of Orthodontics, MGM Dental College and Hospital, Mumbai, Maharashtra, India

${ }^{4}$ Department of Orthodontics, Bharati Vidyapeeth Dental College and Hospital, Mumbai, Maharashtra, India

Corresponding Author: Astha Namdhari, Postgraduate Student, Department of Orthodontics, MGM Dental College and Hospital, Gwalior, Madhya Pradesh, India, e-mail: asthared@yahoo.in
}

Types of Ceramic Brackets: A SEM Study. J Contemp Dent 2018;8(3):125-134.

\section{Source of support: Nil}

Conflict of interest: None

\section{INTRODUCTION}

In 1980s ceramic brackets were introduced because of high expectations of patients for esthetic and less visible appliances. Since then, to evaluate clinical characteristics and properties, a lot of research have been conducted for ceramic brackets. ${ }^{1}$

Different types of ceramic brackets have been manufactured to meet the demands of adults who seek orthodontic treatment. ${ }^{2}$ one of the advantages of ceramic brackets is fewer chances of staining and also less frequency of slot distortion, but the disadvantage is these brackets are very brittle and rigid too. ${ }^{3}$ These features lead to partial or complete bracket fracture due to the pressure of debonding on the base of the bracket. Enamel cracks and fractures have been reported after removal of brackets. ${ }^{4}$

Avoiding such iatrogenic harm to the surface of enamel has been a constant challenge even with the use of metal brackets. Since then, attempts have been made to develop debonding methods that cause minimal undesirable changes in the enamel structure. ${ }^{5}$ There are various techniques for modification of enamel surface and protocols have been described for etching and bonding to reduce the damage to enamel following bracket removal. ${ }^{6-9}$

Planned studies involving various aspects of enamel microcracks (EMCs): The region of their occurrence, the propensity of particular region of the tooth they occur in, whether the cracks are more along mesiodistal width or more along the occlusogingival height, the depth or width of the aforementioned cracks are some of the factors which need to be studied. All of the aforesaid needs to be studied for various types of commercially available ceramic brackets as well as known variations in the debonding techniques.

Micro-cracks in the enamel following debonding is a concern for many patients seeking orthodontic treat- 
ment. ${ }^{10}$ It has been studied that EMCs may jeopardize the integrity of the enamel and cause stains and plaque accumulation on the fractured surfaces. ${ }^{11-13}$ Pronounced Enamel cracks which can be visualized with the naked eye are pronounced micro-cracks and are often easily notified by patients either at the start of the treatment or following removal of the fixed appliances. ${ }^{14,15}$ Current information shows that pronounced EMC characteristics (width and length of EMCs) are more when compared with weak EMCs (not visible under normal illumination but visible under SEM). ${ }^{14-16}$

A modified and economical technique has been introduced recently. This technique ${ }^{17}$ claimed to have a better and safe technique for debonding. In this article describing the technique, authors advocated a fracture line by abrading the bracket body along its long axis between the two tie wings so that the adhesive material stays on the surface of the tooth and the bracket can be removed by squeezing and fracture the tie wings.

The present study targeted to compare and evaluate the occurrence of enamel micro-cracks before bonding and after debonding with monocrystalline ceramic brackets. The brackets used in this study belonged to Ormco (Inspire Ice) and American Orthodontics (Radiance) which were subdivided on the basis of creating a groove (scored and unscored) in these brackets.

The present study was expected to help in evolving a better technique of debonding offered by various types of ceramic brackets tested. The results obtained of this study were expected to refine the esthetic needs without compromising the integrity of enamel when using the monocrystalline ceramic brackets.

\section{MATERIALS AND METHODS}

\section{Materials}

\section{Equipment}

- Scanning electron microscope

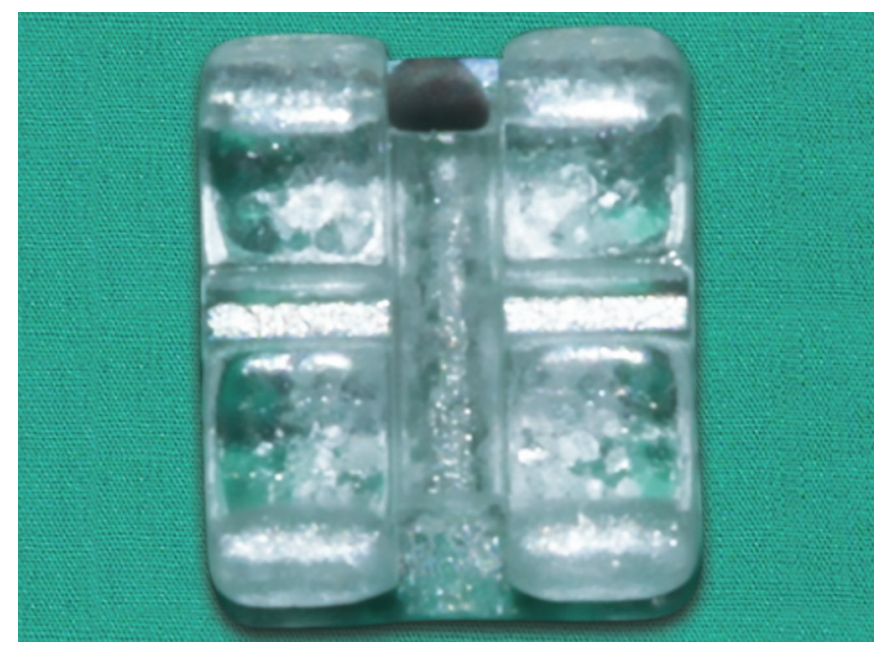

Fig. 1: AO ceramic bracket

\section{Orthodontic Materials}

- Monocrystalline ceramic brackets (AO, USA), 0.022" x 0.028 " slot prescription (Fig. 1)

- Monocrystalline ceramic brackets (Ormco, Europe) $0.022^{\prime \prime}$ x 0.028" slot prescription (Fig. 2)

- Etchant gel (PROetch, SS White) (Fig. 3)

- Adhesive primer (Transbond XT, 3M Unitek ${ }^{\mathrm{TM}}$, USA) (Fig. 4)

- Adhesive paste (Transbond XT, 3M Unitek ${ }^{\mathrm{TM}}$, USA) (Fig. 5)

\section{Extracted Human Premolar Teeth}

Group A: Twenty teeth were bonded with monocrystalline AO ceramic brackets.

Group B: Twenty teeth were bonded with monocrystalline Ormco ceramic brackets.

\section{Orthodontic Instruments}

- Debonding plier (Denticon, India) (Fig. 6)

- Air-turbine (NSK, Japan) (Fig. 7)

- Diamond straight fissure bur (Medicept, TC-11, UK) (Fig. 8)

- Bracket holder (Denticon, India) (Fig. 9)

- LED curing light (Woodpecker, Mini LED, India) (Fig. 10)

- Carbide composite finishing bur, 12 fluted bur (PRIMA CLASSIC, India) (Fig. 11)

- Boone's gauge (3M Unitek ${ }^{\mathrm{TM}}$, USA) (Fig. 12)

\section{Methodology}

Selection Criteria

(A) Inclusion Criteria:

a. Teeth

- Maxillary 1st premolars

- Freshly extracted teeth

- Teeth without fracture

- Noncarious teeth

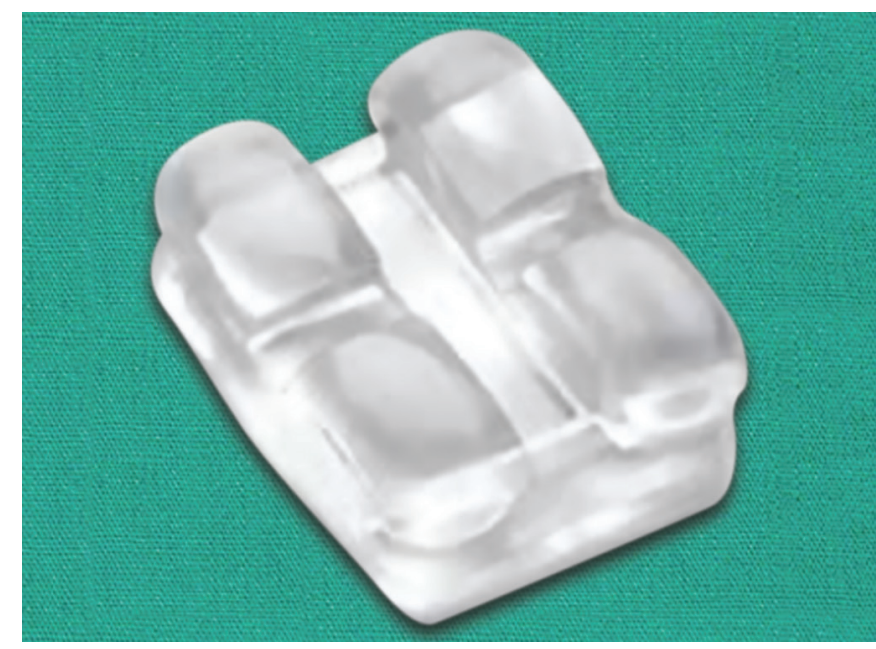

Fig. 2: Ormco ceramic bracket 


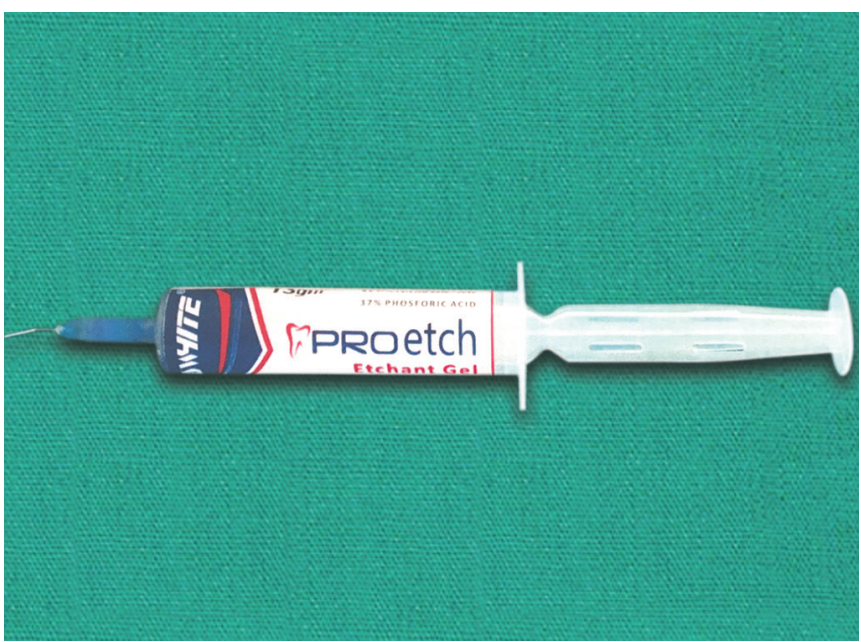

Fig. 3: PROetch Etchant gel (SS White)

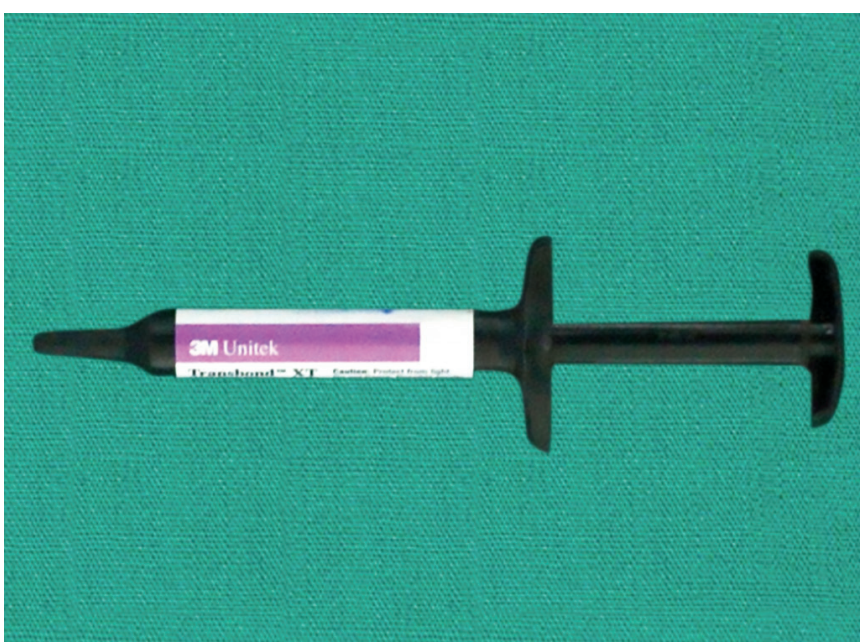

Fig. 5: Adhesive paste: Transbond ${ }^{\mathrm{TM}} \mathrm{XT}$ (3M USA)

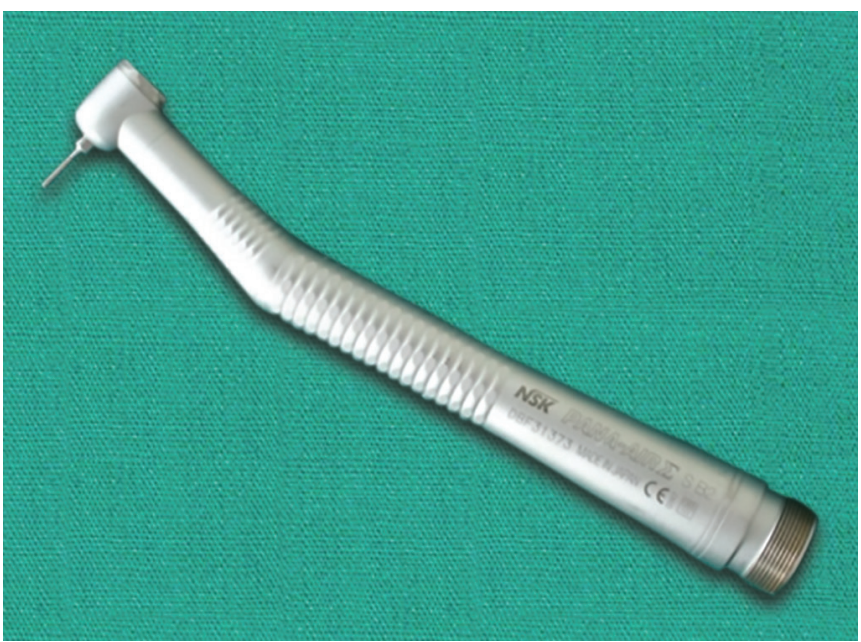

Fig. 7: Airotor handpiece (NSK)

b. Orthodontic Products

- Monocrystalline Ceramic Brackets (AO) 0.022" x 0.028" slot prescription

- Monocrystalline Ceramic Brackets (Ormco) 0.022" x $0.028^{\prime \prime}$ slot prescription

(B) Exclusion Criteria

a. Teeth

- Teeth other than maxillary 1st premolars

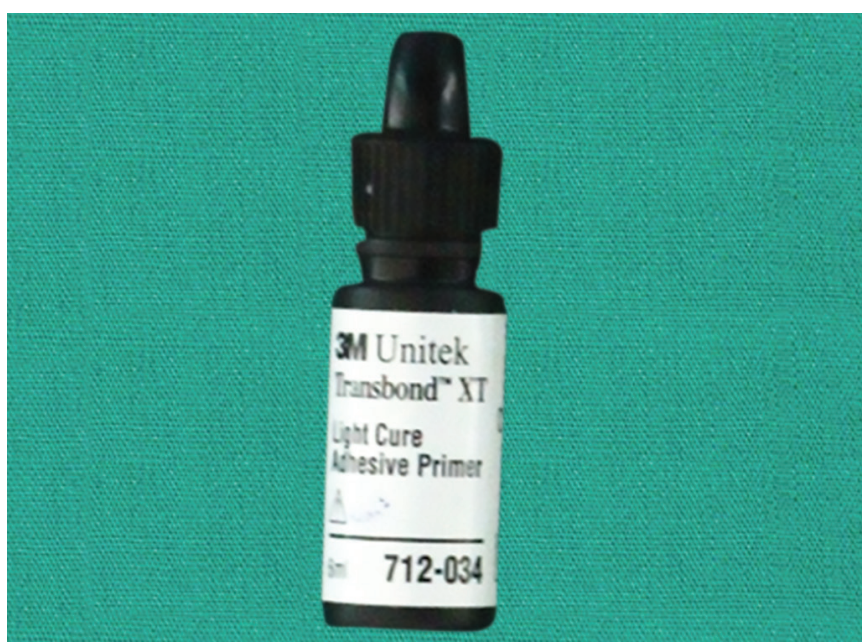

Fig. 4: Adhesive primer: Transbond ${ }^{\mathrm{TM}} \mathrm{XT}(3 \mathrm{M}, \mathrm{USA})$

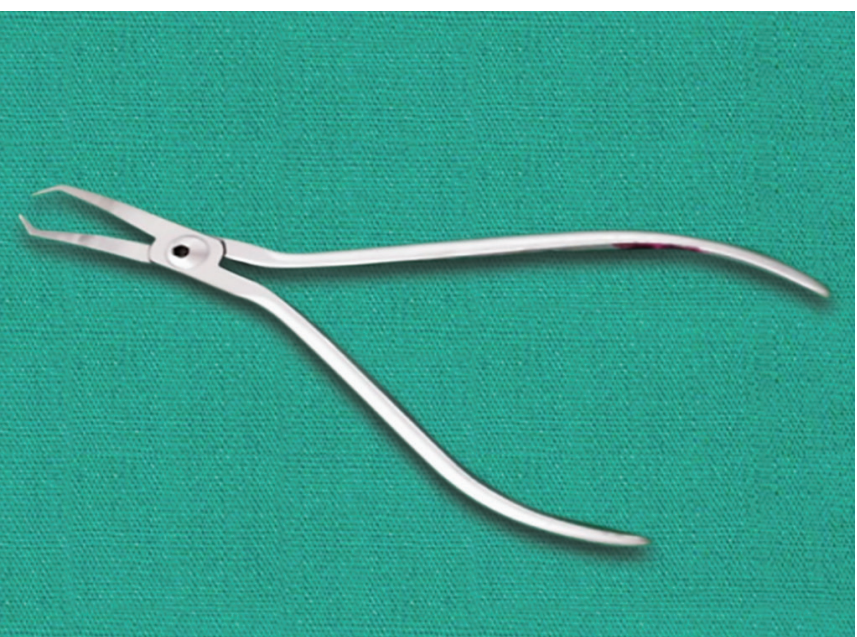

Fig. 6: Debonding plier (Denticon)

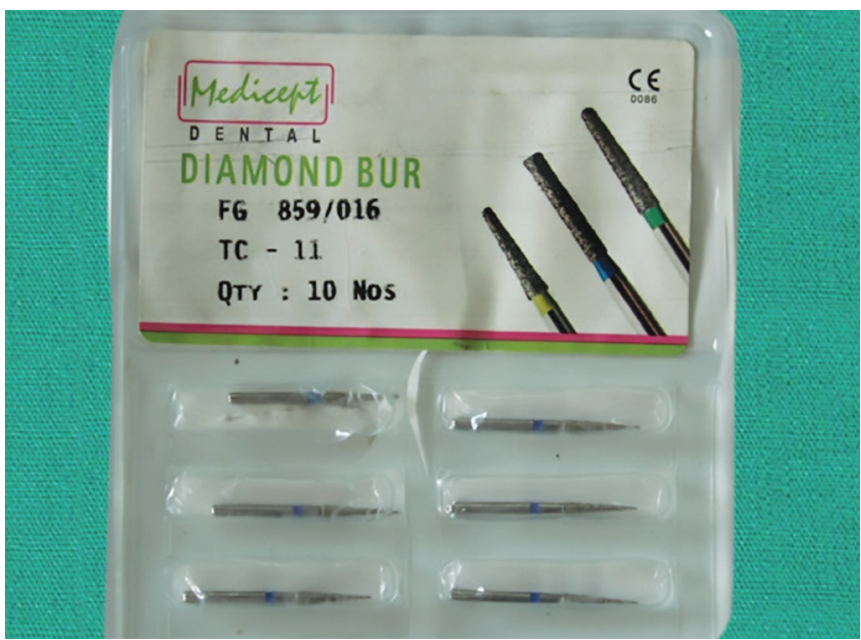

Fig. 8: Tapered fissure diamond bur (Medicept Dental, India)

- Carious teeth

- Fractured teeth

- Stored in chemical agents

b. Orthodontic Products

- Brackets other than AO 0.022" x 0.028" slot prescription

- Brackets other than Ormco 0.022" x 0.028" slot prescription 


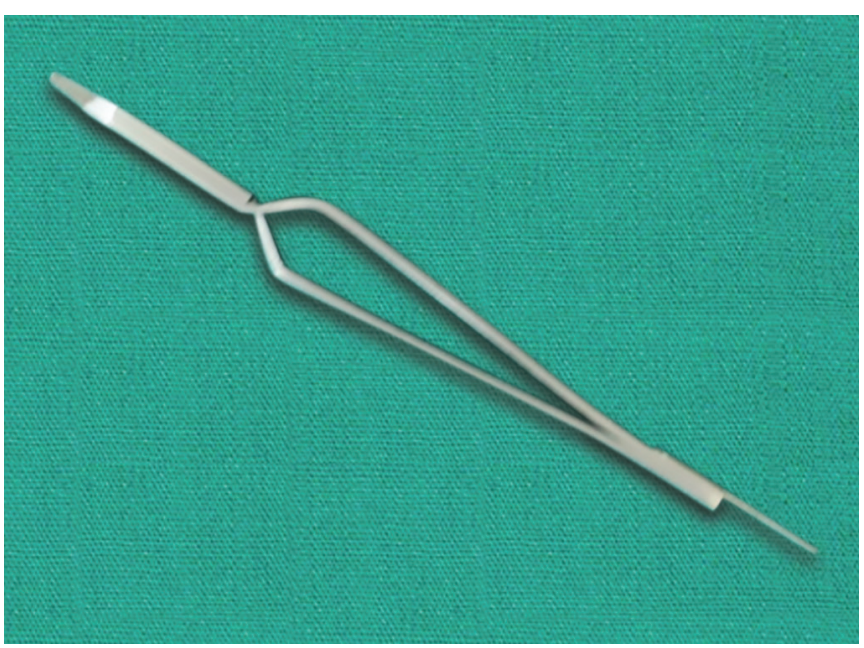

Fig. 9: Bracket holder (Denticon)

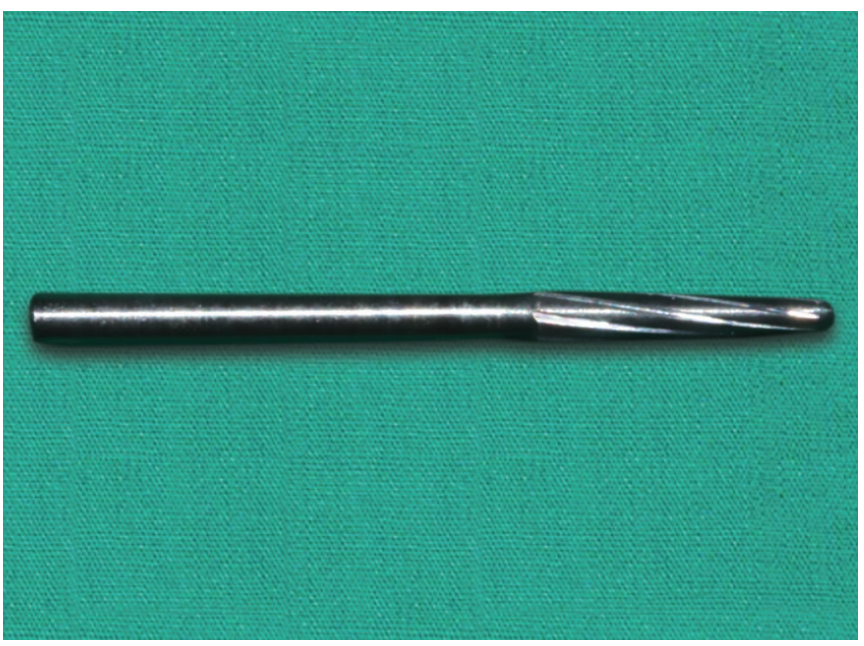

Fig. 11: Carbide finishing bur (Prime Classic, Dental)

\section{Methods of Study}

Extracted forty upper first premolars were used as samples in the study. $0.5 \%$ chloramine solution was used to decontaminate the samples. Enamel on buccal surfaces of teeth was evaluated with the help of SEM. The microscope was operated at $15 \mathrm{kV}$ and $100 \mathrm{x}$ was used to evaluate the enamel micro-cracks initially (Fig. 13).

Samples were divided into two groups of 20 after examining with the scanning electron microscope:

Group A: AO monocrystalline ceramic brackets Group B: Ormco monocrystalline ceramic brackets Groups A and B were again subdivided into four groups of 10 :

Group $A_{1}$ : AO monocrystalline ceramic brackets (scored) Group $_{2}$ : AO monocrystalline ceramic brackets (unscored) Group $B_{1}$ : Ormco monocrystalline ceramic brackets (scored)

Group $B_{2}$ : Ormco monocrystalline ceramic brackets (unscored)

The teeth were etched with phosphoric acid (3M, Unitek) for 30 seconds, rinsed for 20 seconds with water,

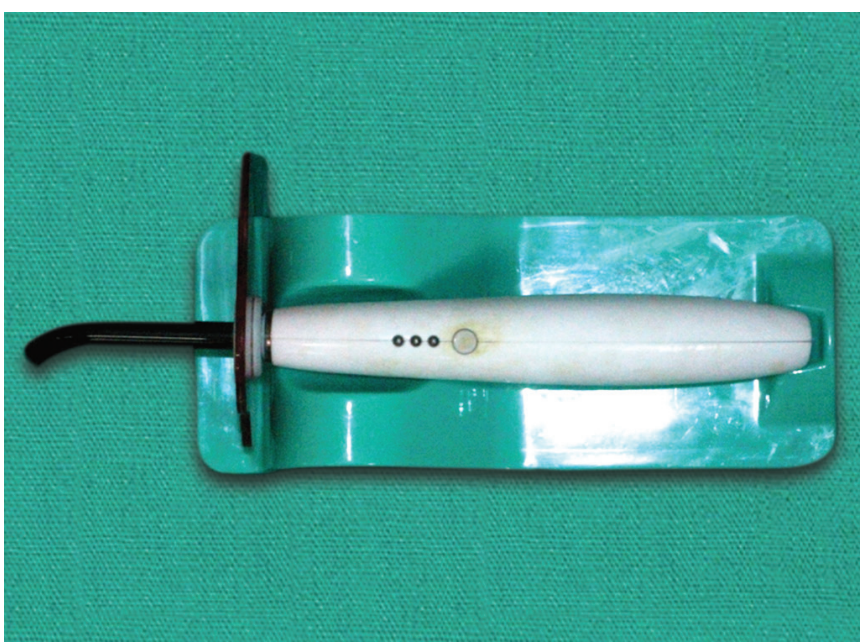

Fig. 10: Light cure unit (Woodpecker)

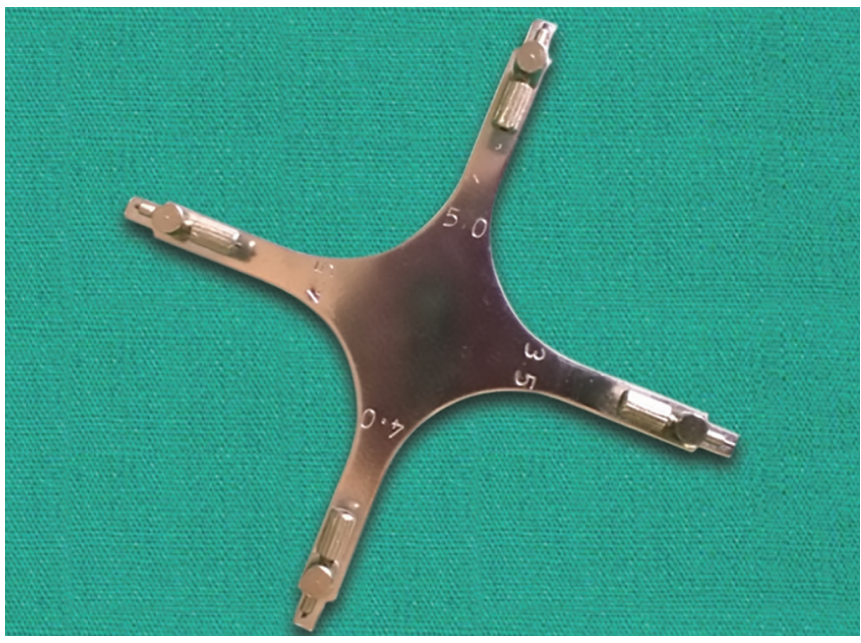

Fig. 12: Boone's gauge (3M, Unitek)

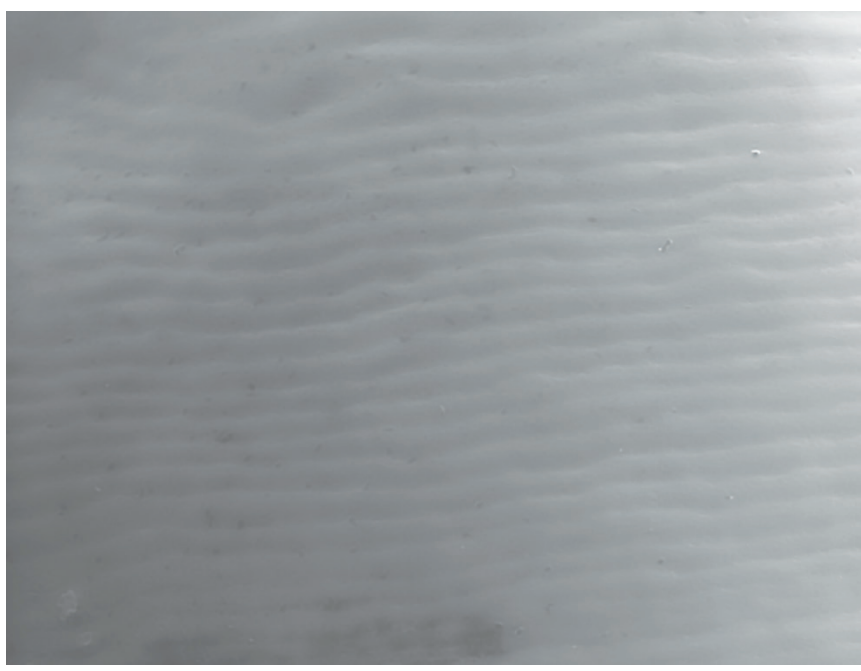

Fig. 13: Sample specimen under SEM before bonding showing no cracks under $100 \mathrm{x}$

then dried for 10 seconds with air. After that, primer (3M Unitek) was applied with an applicator (Fig. 14) and curing was done for 20 seconds. Resin adhesive (Transbond XT, 3M Unitek, USA) was applied to the base of the ceramic bracket. Then, bracket holder (Denticon) 


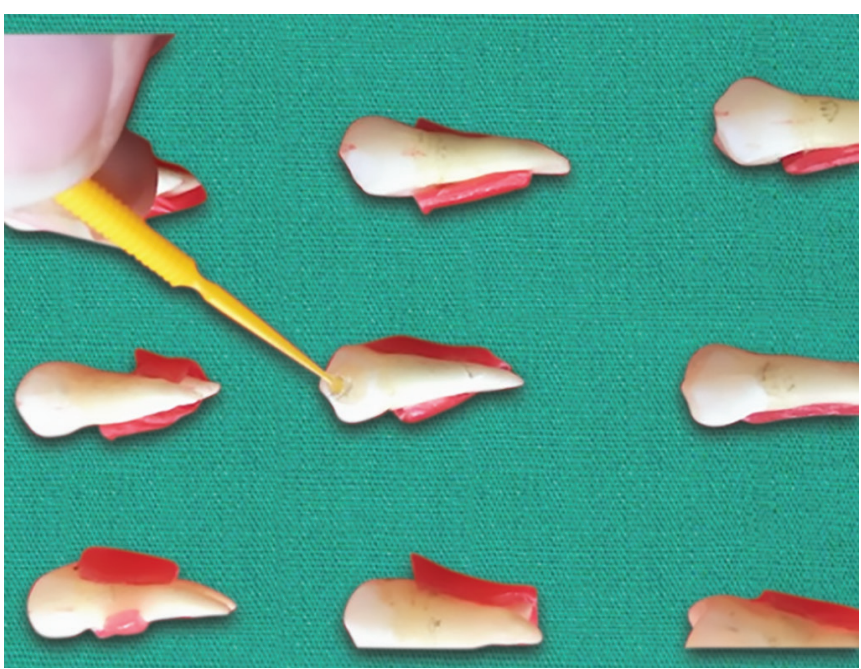

Fig. 14: Application of bonding agent on a sample

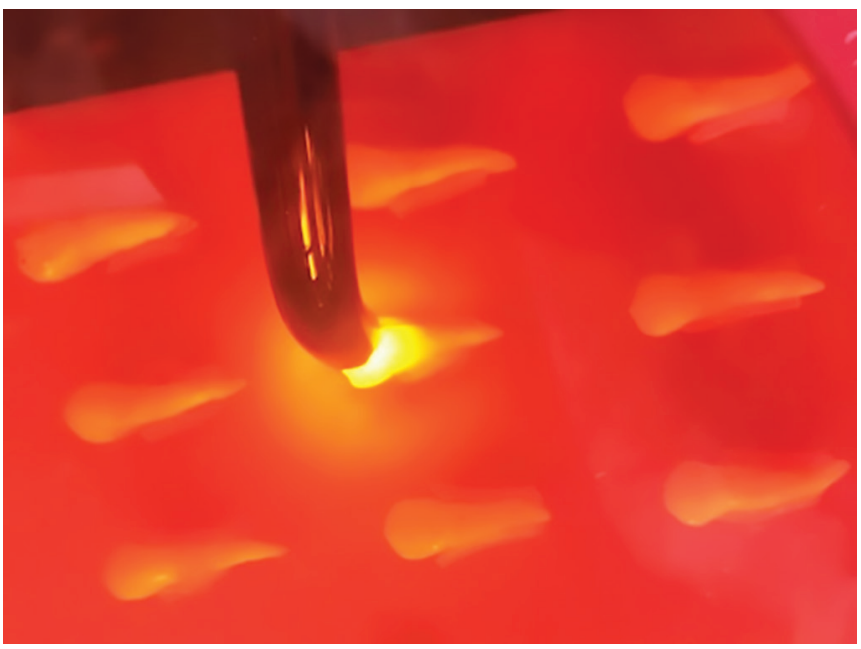

Fig. 16: Photopolymerization of bracket

was used to firmly position the bracket on the surface of enamel (Fig. 15) and explorer was used to remove the excess adhesive around the bracket base. Halogen light (Mini L.E.D, Woodpecker) was used to polymerize the adhesive (Fig. 16). After this procedure, distilled water was used to store the teeth at $37^{\circ} \mathrm{C}$ before testing for 24 hours. The average base area for maxillary premolar monocrystalline ceramic brackets (Ormco) was 11.83 $\mathrm{mm}^{2}$.

In this study, the 'search area' was located at the very center of every tooth. This search area was used to coincide with the bracket base region. This center of the tooth surface was marked around the bracket base by a permanent marker (Fig. 17).

For scoring Group $\mathrm{A}_{1}$ and Group $\mathrm{B}_{1}$, a fracture line was created by abrading the bracket body along its long axis between the two tie wings (Fig.18). Long, tapered, high-speed diamond bur (Medicept, TC-11, UK) was used to cut down the bracket into two pieces till the adhesive layer according to the diameter of bur (Fig. 19). After

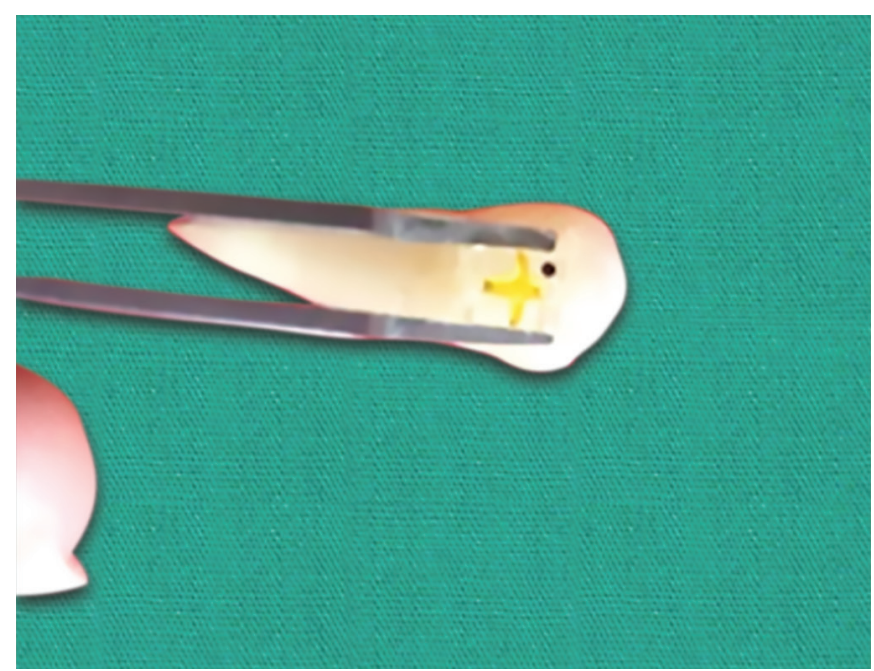

Fig 15: Bracket placement

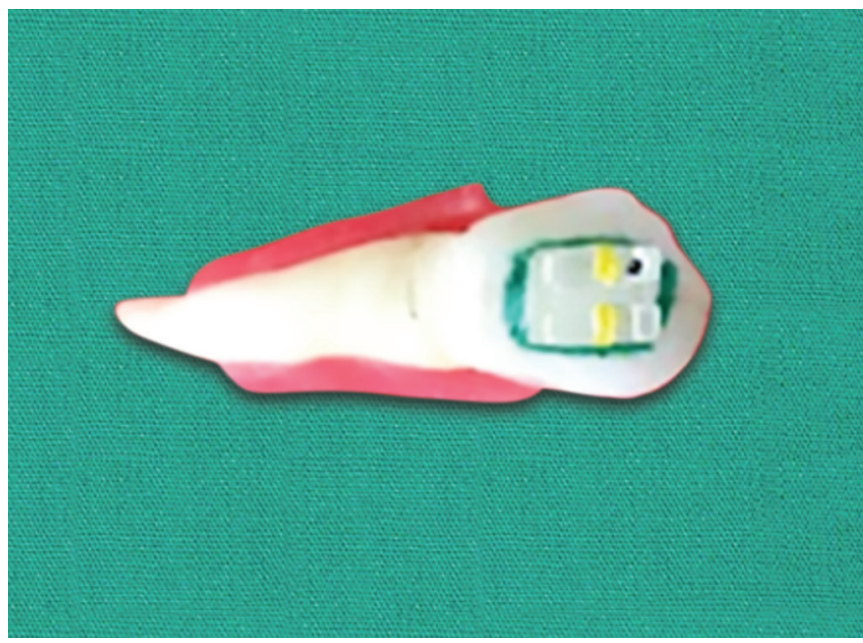

Fig. 17: Marking around bracket boundary with a marker

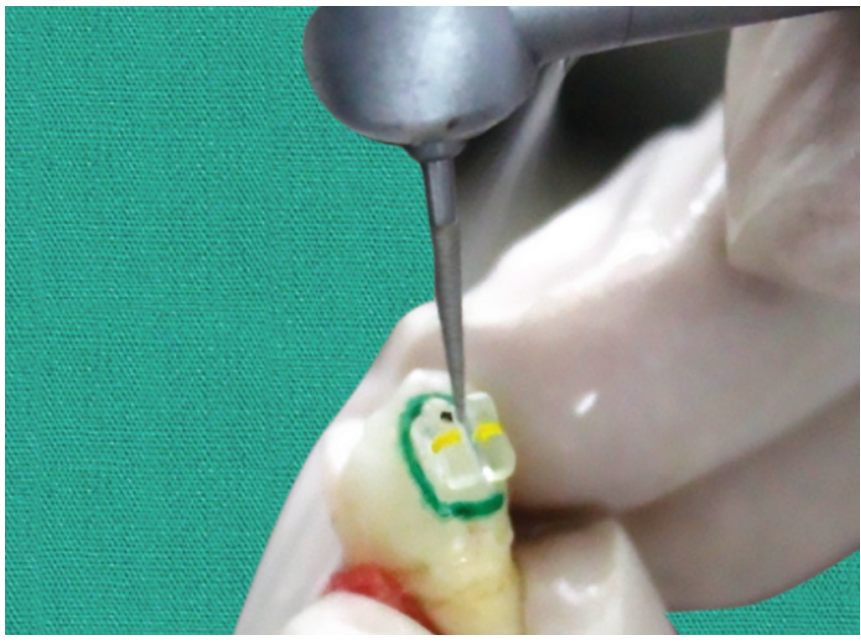

Fig 18: Scoring of ceramic bracket

creating the fracture line, debonding plier was used to squeeze and fracture the tie wings (Fig. 20).

After removal of brackets (Fig. 21), ARI was recorded on each tooth and adhesive remnants were removed by low-speed, 12 fluted safe end carbide bur. 


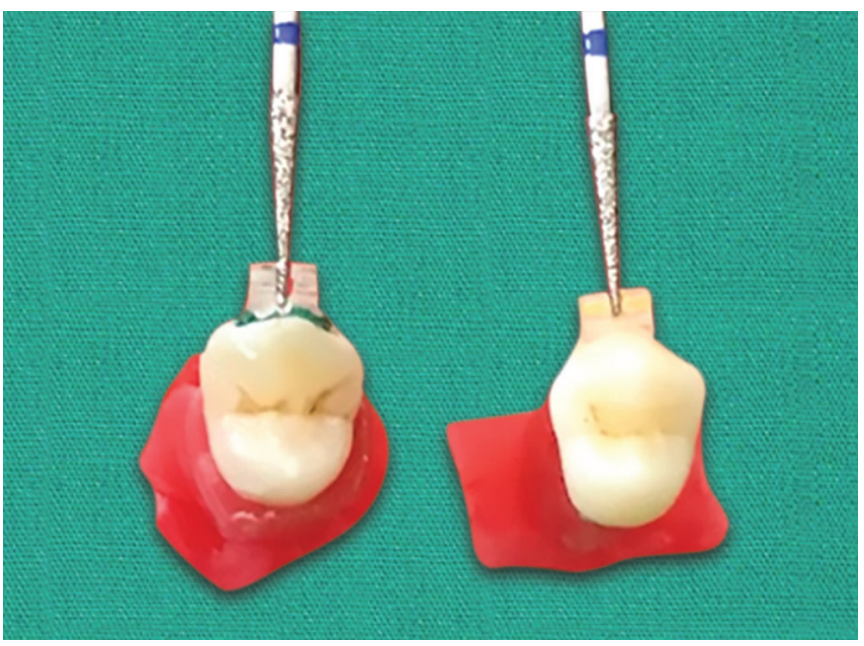

Fig. 19: Comparison between scored and unscored bracket

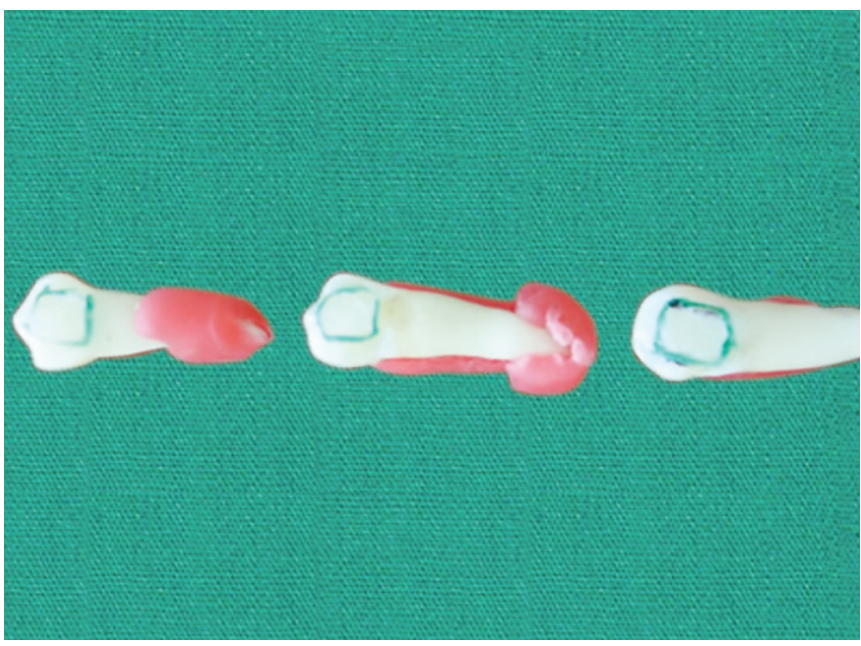

Fig. 21: Samples after debonding

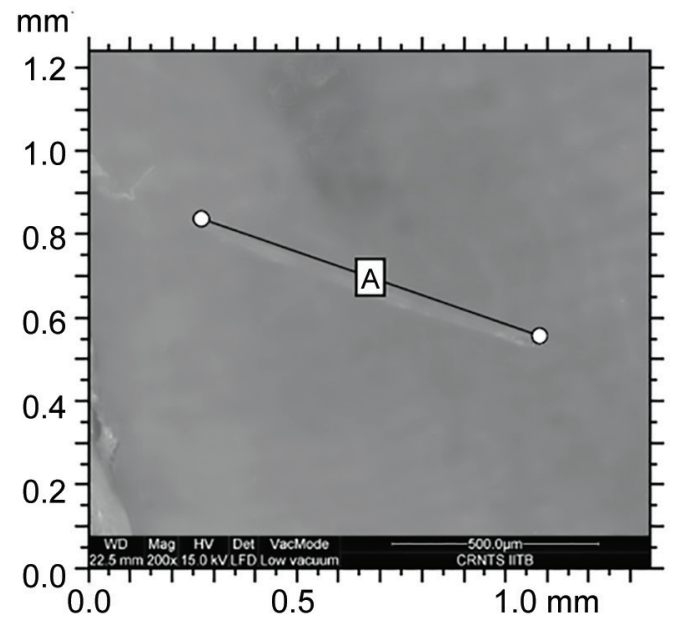

Fig. 23: Measurement of length of enamel micro-crack of a sample under $200 x$

Qualitative and quantitative micro-crack characteristics were analyzed as a number of micro-cracks and width and length of the largest micro-crack in the enamel. Enamel micro-cracks were visualized under SEM (Fig. 22). The width and length of the micro-crack which was longer was quantified (Figs 23 and 24).

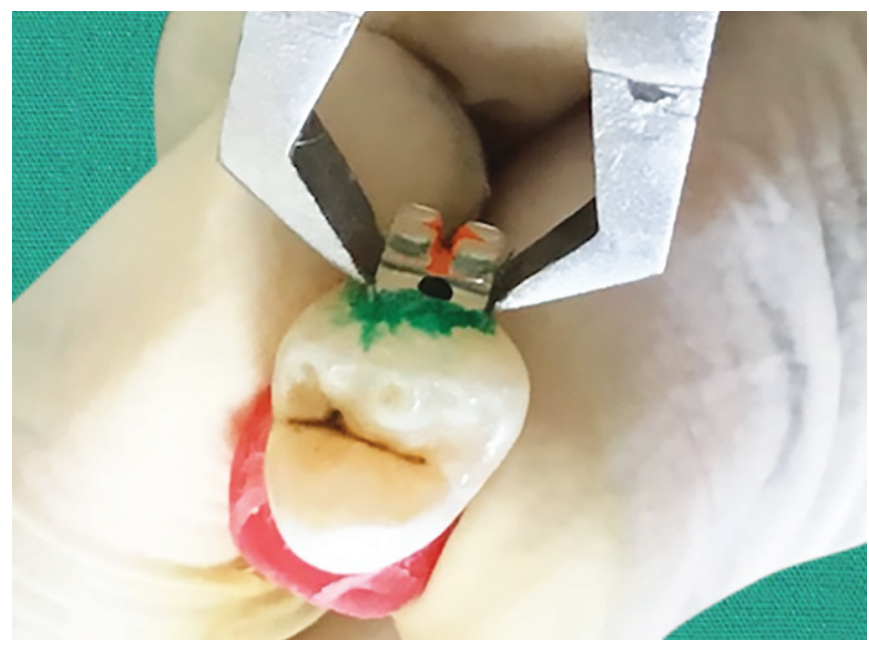

Fig. 20: Debonding of ceramic bracket

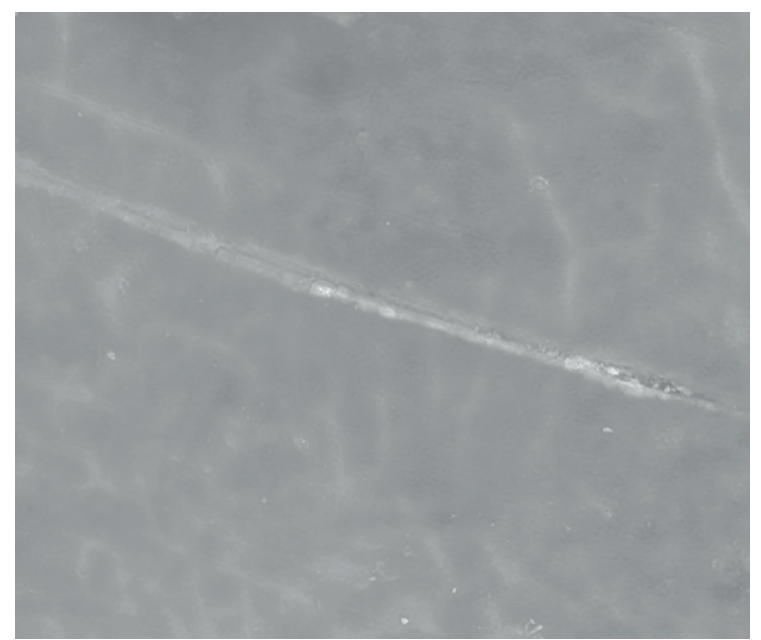

Fig. 22: Sample specimen under SEM after debonding at 400x after debonding showing enamel micro-crack

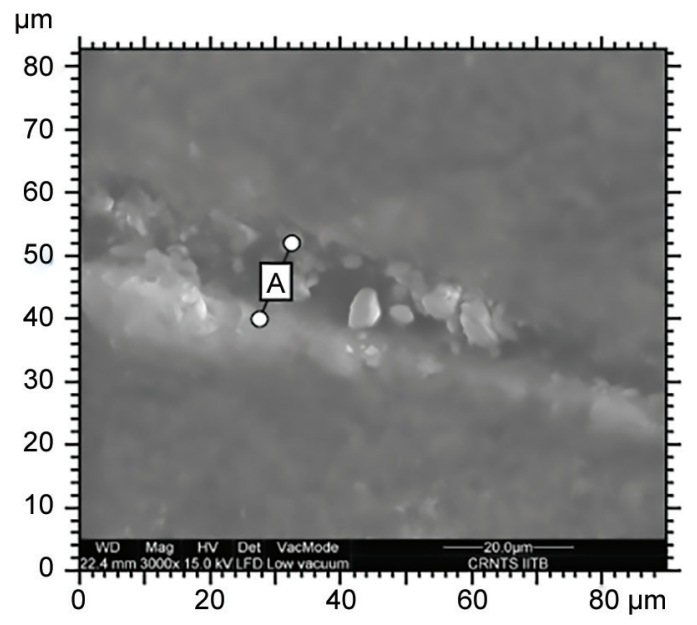

Fig 24: Measurement of width of enamel micro-crack of a sample with enamel micro-crack under $3000 x$

\section{RESULTS}

Results of the study are represented in a tabular and graphic form which are as follows:

Table 1 depicts the difference in the occurrence of enamel micro-cracks between scored monocrystalline ceramic brackets and unscored monocrystalline ceramic 
brackets of both the $\mathrm{AO}$ and Ormco groups. Relative risk was used to measure the association between groups $\left(A_{1}\right.$ and $\left.B_{1}\right)$ and groups $\left(A_{2}\right.$ and $\left.B_{2}\right)$. This difference was not statistically significant $(\mathrm{p}=0.096)$.

Graph 1 depicts a bar chart representing the difference in the percentage of enamel micro-cracks between groups $\left(A_{1}\right.$ and $\left.B_{1}\right)$ and groups $\left(A_{2}\right.$ and $\left.B_{2}\right)$. It reveals that enamel micro-cracks developed only in Groups $\left(A_{2}\right.$ and $\left.B_{2}\right)$ and cracks are absent in Groups $\left(\mathrm{A}_{1}\right.$ and $\left.\mathrm{B}_{1}\right)$.

Table 2 is depicting the difference in the occurrence of enamel micro-cracks between groups A and B. Relative risk was used to measure the association between group $A$ and $B$. This difference is not statistically significant ( $p$ $=0.096)$.

Graph 2 depicts a bar chart representing the difference in the percentage of enamel micro-cracks between

Table 1: Comparison of enamel micro-cracks among scored and unscored brackets

\begin{tabular}{lllc}
\hline & \multicolumn{2}{l}{ Scoring of brackets } & \\
\cline { 2 - 3 } Enamel & Scored & Unscored & \\
cracks & (Groups & (Groups & \\
observed & A1 and & A2 and & \\
\hline Absent & 20 & B2) & $35(87.5 \%)$ \\
Present & 0 & 15 & $5(12.5 \%)$ \\
& 20 & 5 & 40 \\
\hline
\end{tabular}

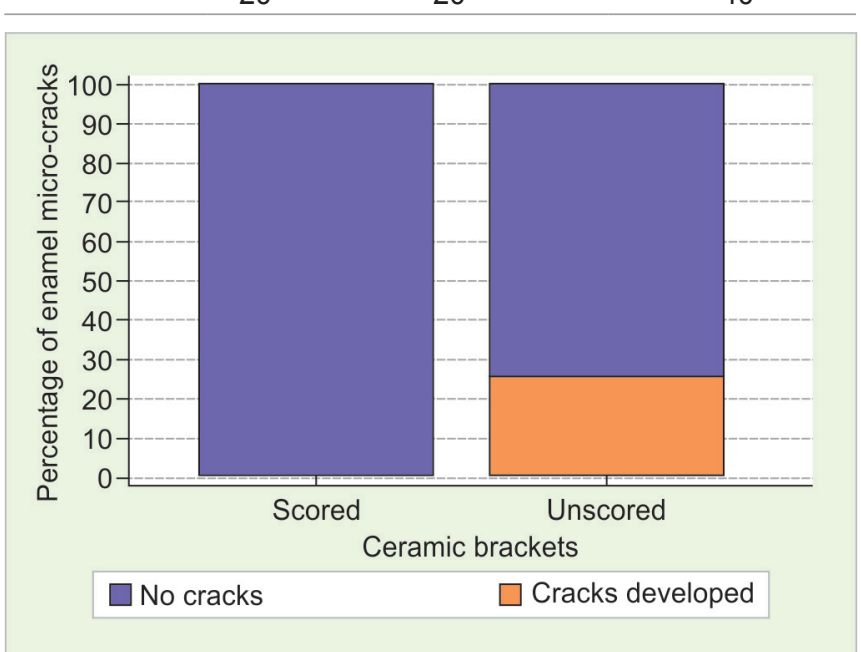

Graph 1: Comparison of enamel micro-cracks among scored and unscored brackets
Group B and Group A. It reveals that the percentage of enamel micro-cracks is less in group B than Ggroup A.

Table 3 is depicting the relation between the scoring of ARI index and development of enamel micro-cracks. Chi-square test is performed to know whether there is a significant difference between the scoring of ARI and enamel micro-cracks development. This difference is found which is significant statistically ( $p=0.013$ ).

Graph 3 depicts the relation between ARI scoring and percentage of enamel micro-cracks. A number of enamel micro-cracks are more in samples having less score.

Tables 4 and 5 are depicting the width and length of each micro-crack in the enamel of groups $\left(A_{2}\right.$ and $\left.B_{2}\right)$.

Table 6 is comparing the mean, standard deviation, standard error and confidence interval of the length of enamel micro-cracks between groups $\left(A_{2}\right.$ and $\left.B_{2}\right)$. The

Table 2: Comparison of enamel micro-cracks among scored and unscored brackets

\begin{tabular}{lllc}
\hline $\begin{array}{l}\text { Enamel } \\
\text { Cracks } \\
\text { observed }\end{array}$ & $\begin{array}{l}\text { Brackets } \\
\text { American Orthodontics }\end{array}$ & $\begin{array}{l}\text { Ormco } \\
\text { (Group A) }\end{array}$ & \\
\hline Absent & 17 & 18 & $35(87.5 \%)$ \\
Present & 3 & 2 & $5(12.5 \%)$ \\
& 20 & 20 & 40 \\
\hline
\end{tabular}

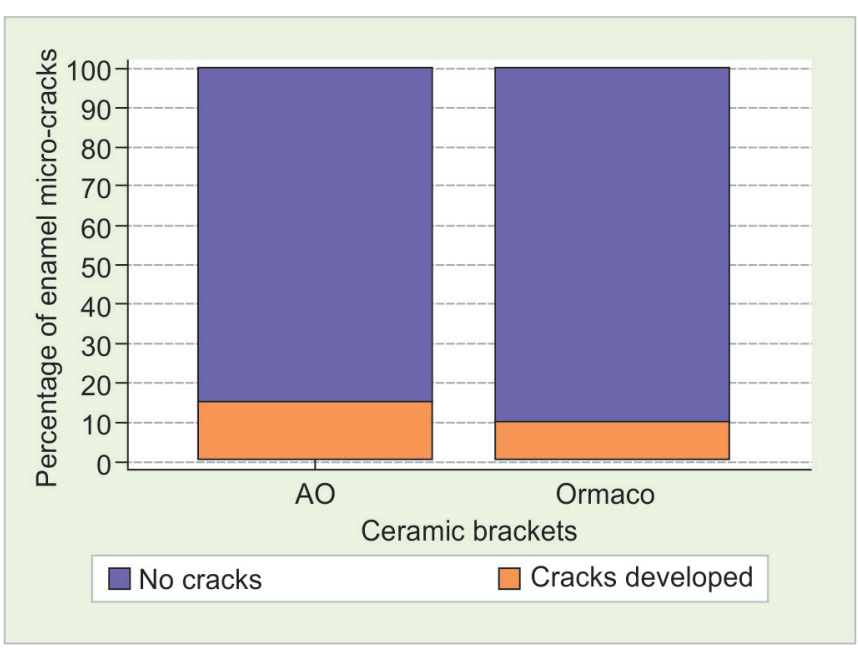

Graph 2: Comparison of enamel micro-cracks among American orthodontic and Ormco brackets

Table 3: Relationship between ARI scoring and development of enamel micro-cracks

\begin{tabular}{|c|c|c|c|c|c|c|}
\hline \multirow[b]{2}{*}{ Cracks } & \multicolumn{6}{|l|}{$A R I$} \\
\hline & No Adhesive & $\begin{array}{l}<25 \% \\
\text { adhesive left }\end{array}$ & $\begin{array}{l}25-50 \% \\
\text { adhesive left }\end{array}$ & $\begin{array}{l}50-75 \% \\
\text { adhesive left }\end{array}$ & $\begin{array}{l}75-100 \% \\
\text { adhesive left }\end{array}$ & $\begin{array}{l}100 \% \text { adhesive } \\
\text { left }\end{array}$ \\
\hline \multirow[t]{4}{*}{ Absent } & 2 & 2 & 7 & 10 & 8 & 6 \\
\hline & $5.7 \% \mathrm{RT}$ & $5.7 \% \mathrm{RT}$ & $20.0 \%$ RT & $28.6 \%$ RT & $22.9 \%$ RT & $17.1 \% \mathrm{RT}$ \\
\hline & $50.0 \%$ CT & $40.0 \%$ CT & $100.0 \%$ CT & $100.0 \% \mathrm{CT}$ & $100.0 \%$ CT & $100.0 \%$ CT \\
\hline & $5.0 \% \mathrm{GT}$ & $5.0 \% \mathrm{GT}$ & $17.5 \%$ GT & $25.0 \%$ GT & $20.0 \%$ GT & $15.0 \%$ GT \\
\hline \multirow[t]{6}{*}{ Present } & 2 & 3 & 0 & 0 & 0 & 0 \\
\hline & $40.0 \%$ RT & $60.0 \%$ RT & $0.0 \% \mathrm{RT}$ & $0.0 \% \mathrm{RT}$ & $0.0 \% \mathrm{RT}$ & $0.0 \% \mathrm{RT}$ \\
\hline & $50.0 \%$ CT & $60.0 \% \mathrm{CT}$ & $0.0 \% \mathrm{CT}$ & $0.0 \% \mathrm{CT}$ & $0.0 \% \mathrm{CT}$ & $0.0 \% \mathrm{CT}$ \\
\hline & $5.0 \%$ GT & $7.5 \%$ GT & $0.0 \% \mathrm{GT}$ & $0.0 \% \mathrm{GT}$ & $0.0 \%$ GT & $0.0 \% \mathrm{GT}$ \\
\hline & 4 & 5 & 7 & 10 & 8 & 6 \\
\hline & $(10.0 \%)$ & $(12.5 \%)$ & $(17.5 \%)$ & $(25.0 \%)$ & $(20.0 \%)$ & $(15.0 \%)$ \\
\hline
\end{tabular}




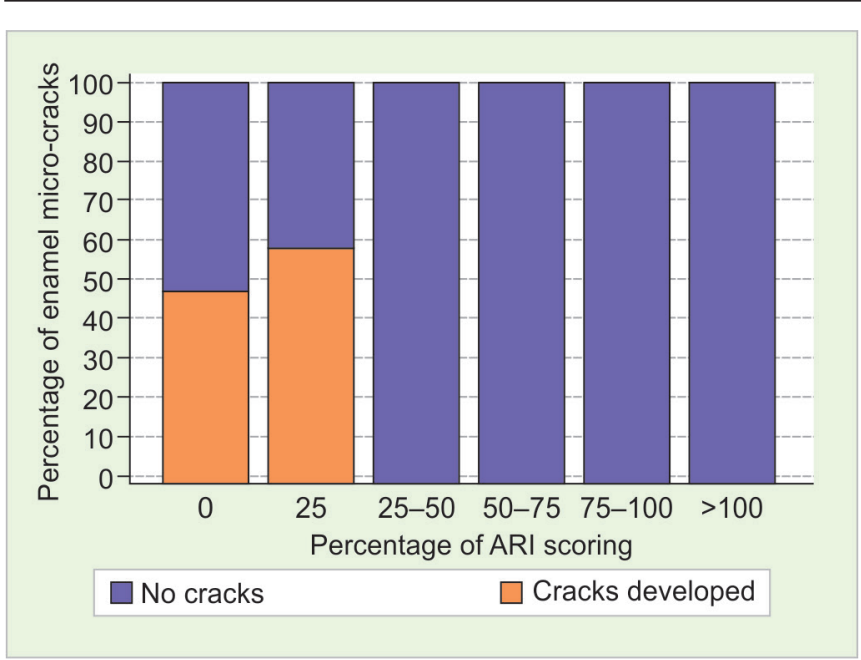

Graph 3: Relationship between ARI scoring and development of enamel microcracks

difference of length between the 2 groups is statistically insignificant $(\mathrm{p}=0.77)$

Graph 4 depicts a bar chart representing the mean length of micro-cracks in the enamel in both the $\mathrm{AO}$ and Ormco ceramic brackets.

Table 7 is comparing the mean, standard deviation, standard error and confidence interval of the width of enamel micro-cracks between $\mathrm{AO}$ and Ormco groups. The difference of width between the 2 groups is statistically insignificant $(\mathrm{p}=0.24)$.

Graph 5 depicts a bar chart representing the mean width of micro-cracks in the enamel in both the $\mathrm{AO}$ and Ormco ceramic brackets.

\section{DISCUSSION}

In the treatment of adult patients, the addition of esthetic brackets was a much-celebrated event. It has been accepted by adults which has expanded the development of contemporary orthodontic modalities. Since their

Table 4: Length and width of each enamel micro-crack of Group $A_{2}$

\begin{tabular}{lll}
\hline Samples & Length of cracks & Width of cracks \\
\hline 1 & $0.8 \mathrm{~mm}$ & $13 \mu \mathrm{m}$ \\
2 & $0.5 \mathrm{~mm}$ & $10.8 \mu \mathrm{m}$ \\
3 & $1.16 \mathrm{~mm}$ & $17.16 \mu \mathrm{m}$ \\
\hline
\end{tabular}

Table 6: Descriptive statistics of length of enamel micro-cracks

\begin{tabular}{lll}
\hline & Group $A_{2}$ & Group $B_{2}$ \\
\hline Sample size & 3 & 2 \\
Arithmetic mean & 0.8200 & 0.8700 \\
95\% Cl for & -0.0008938 to 1.6409 & -3.8313 to \\
the mean & & 5.5713 \\
Lowest value & 0.5000 & 0.5000 \\
Highest value & 1.1600 & 1.2400 \\
Median & 0.8000 & 0.8700 \\
Variance & 0.1092 & 0.2738 \\
Standard deviation & 0.3305 & 0.5233 \\
Standard error & 0.1908 & 0.3700 \\
of the mean & & \\
\hline
\end{tabular}

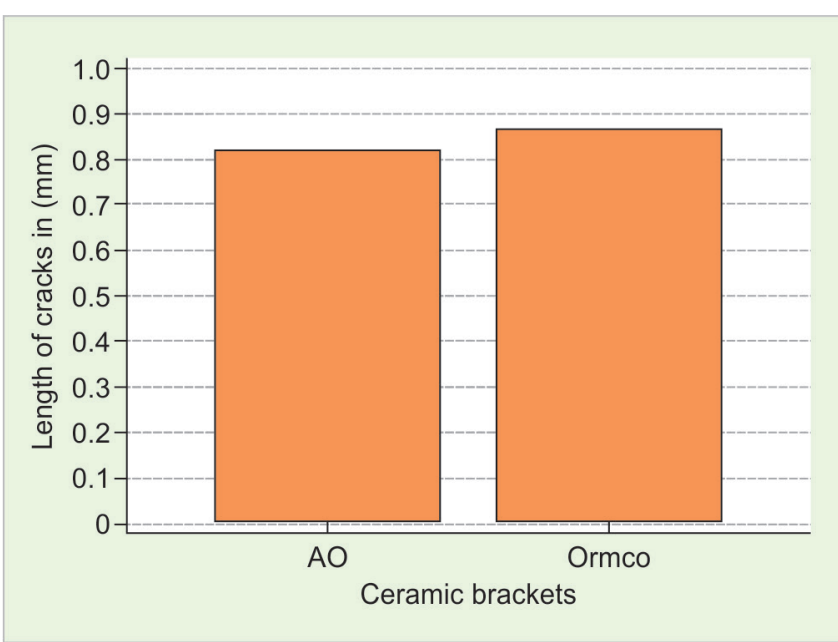

Graph 4: Mean length of enamel microcracks in both the ceramic brackets

advent, progressive improvements in product design have been noted which has resulted in the superior performance of ceramic brackets.

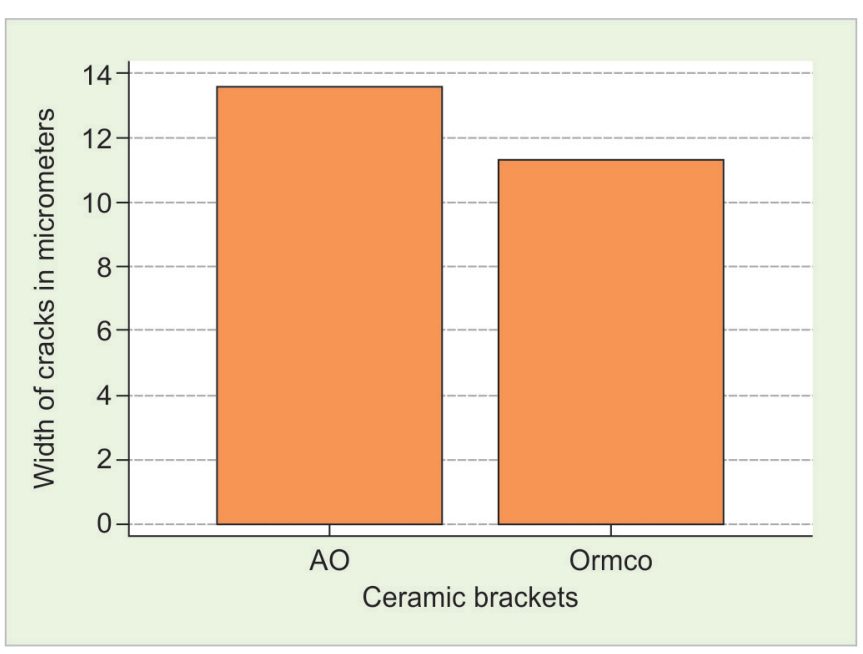

Graph 5: Mean width of enamel microcracks in both the ceramic brackets

Table 5: Length and width of each enamel micro-crack of Group $B_{2}$

\begin{tabular}{lll}
\hline Samples & Length & Width \\
\hline 1 & $1.24 \mathrm{~mm}$ & $12.3 \mu \mathrm{m}$ \\
2 & $0.5 \mathrm{~mm}$ & $10.5 \mu \mathrm{m}$ \\
\hline
\end{tabular}

Table 7: Descriptive statistics of width of enamel micro-cracks

\begin{tabular}{lll}
\hline & Group $A_{2}$ & Group $B_{2}$ \\
\hline Sample size & 3 & 2 \\
Arithmetic mean & 13.6533 & 11.4000 \\
95\% Cl for the mean & 5.6297 to 21.6770 & -0.03558 to 22.8356 \\
Lowest value & 10.8000 & 10.5000 \\
Highest value & 17.1600 & 12.3000 \\
Median & 13.0000 & 11.4000 \\
Variance & 10.4325 & 1.6200 \\
Standard deviation & 3.2299 & 1.2728 \\
Standard error & 1.8648 & 0.9000 \\
of the mean & & \\
\hline
\end{tabular}


Damage to the enamel occurring due to the removal of ceramic brackets has been the concern to many researchers and has a number of studies. Some of these studies have evaluated many factors related to bonding procedure such as the etching time and the type of resin adhesive used. ${ }^{18,19}$ Some have assessed the types of bracket bases, having different groove designs $\mathrm{s}^{20-22}$ or mechanism for retentions of the bonding agents. Many studies evaluated bonding method, bonding material or combination of both.

"Primum non nocere", the phrase from Hippocratic Oath, means "Do no harm". In healthcare sciences, the aim of designing any treatment method is to avoid iatrogenic damage at any cost. Reducing the occurrence of microcracks at the enamel surface is essential to maintain the pretreatment status of the tooth surface. In this context, one of the major goals of orthodontic practitioners is the search for a technique that would have no harmful effects on the teeth. Over the last few decades, major efforts have been made to develop an efficient debonding for ceramic brackets. However, Silveira, Mucha, and Bittencourt ${ }^{17}$ demonstrated a technique to provide a safe technique of bracket removal for ceramic brackets.

Studies that evaluate the debonding produced by different brackets have a great diversity. This is because of the variety of techniques used. Tensile bond failure occurs at the bracket-adhesive interface when squeezing pressure is applied with debonding plier to remove the ceramic bracket. This is the most effective method to prevent enamel damage. ${ }^{23}$ The disadvantage of this procedure is that it cannot be effectively used when an edgewise full-size wire is in place, and it is unsuitable for reuse. Another mechanical method is to squeeze or twist the bracket at its base, but this method can cause damage to enamel due to rubbing of sharp ends of pliers and also due to the site of failure being adhesive enamel interface.

Ultrasonic method is another method of debonding which is relatively safe, but it is very slow as compared to other debonding methods and requires additional time for removing brackets ${ }^{24}$ and this method is expensive also. ${ }^{25}$ The electrothermal debonding technique is another method of debonding but it has limited application $^{24}$ and it has a potential for pulpal damage. ${ }^{26}$

Silveira, Mucha, and Bittencourt ${ }^{17}$ proposed a simple, safe and economical technique to create a stress concentrator in any brand of the ceramic bracket by scoring it with a diamond bur followed by debonding with a plier. In this technique, presented in a journals technique clinic corner, they suggested creating a fracture line by abrading the bracket body along its long axis between the two tie wings so that the resin stays on the surface of the tooth and the bracket can be removed by squeezing and fracture the tie wings.
We decided to test a similar method as a well-designed study to establish a debonding protocol by statistically evaluate the data collected through laboratory tests performed under loupes and SEM by evaluating the samples debonded using two different methods.

In this study, 40 extracted upper 1st premolars were included. These samples were evaluated under SEM for micro-cracks in enamel before bonding the brackets and after debonding the brackets. There were no enamel micro-cracks before bonding ceramic brackets. Among 40 samples, 20 had bonding with $\mathrm{AO}$ ceramic brackets and 20 had bonding with monocrystalline Ormco ceramic brackets. Among 20 in both groups, 10 were scored with tapered fissure diamond bur. This scoring resulted in shattering of ceramic brackets into different pieces during debonding and left the major amount of the resin on the surface of the tooth.

When testing carried out, scored monocrystalline ceramic brackets produced no enamel micro-cracks while few samples of unscored monocrystalline ceramic brackets produced enamel micro-cracks.

Thus, this study helped in evolving a better technique of debonding for various types of ceramic brackets tested. The results obtained of this study were expected to refine the clinical treatment steps without compromising the integrity of enamel when using the monocrystalline ceramic brackets.

\section{CONCLUSION}

Post debonding, significant difference was not found in the length or width of enamel micro-cracks between $\mathrm{AO}$ monocrystalline ceramic brackets (group A) and Ormco monocrystalline ceramic brackets (group B).

Micro-cracks in enamel found in only Unscored Monocrystalline American Orthodontics (group $\mathrm{A}_{2}$ ) and unscored monocrystalline ormco (Group $\mathrm{B}_{2}$ ) ceramic Brackets.

With high ARI index, there is decreased enamel microcracks seen in both american orthodontics (Group A) and ormco (Group B) ceramic brackets.

\section{REFERENCES}

1. Kitahara-Céia FMF, Mucha JN, dos Santos PAM. Assessment of enamel damage after removal of ceramic brackets. American Journal of Orthodontics and Dentofacial Orthopedics [Internet]. Elsevier BV; 2008 Oct;134(4):548-555.

2. Theodorakopoulou LP, Sadowsky PL, Jacobson A, Lacefield $\mathrm{Jr}$ W. Evaluation of the debonding characteristics of 2 ceramic brackets: an in vitro study. American Journal of Orthodontics and Dentofacial Orthopedics. 2004 Mar 1;125(3):329-336.

3. Bishara SE, Fehr DE. Ceramic brackets: something old, something new, a review. Semin Orthod 1997;3:178-188.

4. Kusy RP. Morphology of the polycrystalline alumina bracket and its relationship to fracture toughness and strength. Angle Orthod 1988;58:197-203. 
5. Irma Dumbryte, Laura Linkeviciene, Mangirdas Malinauskas, Tomas Linkevicius, Vytaute Peciuliene, Kristupas Tikuisis. Evaluation of enamel cracks characteristics after removal of metal brackets in adult patients Eur J Orthod 2013;35:317-322

6. Shahabi M, Heravi F, Mokhber N, Karamad R, Bishara SE. Effects on shear bond strength and the enamel surface with an enamel bonding agent. Am J Orthod Dentofacial Orthop 2010;137: 375-378.

7. Tecco S, Tetè S, D'Attilio M, Festa F. Enamel surface after debracketing of orthodontic brackets bonded with flowable orthodontic composite. A comparison with a traditional orthodontic composite resin. Minerva Stomatol 2008; 57:81-94.

8. Lamper T, Ilie N, Huth KC, Rudzki I, Wichelhaus A, Paschos E. Self-etch adhesives for the bonding of orthodontic brackets: faster, stronger, safer? Clin Oral Investig 2014;18:313-319.

9. Elekdag-Turk S, Isci D, Ozkalayci N, Turk T. Debonding characteristics of a polymer mesh base ceramic bracket bonded with two different conditioning methods. Eur J Orthod 2009; 31:84-89.

10. Kitahara-Ceia FM, Mucha JN, Marques dos Santos PA. Assessment of enamel damage after removal of ceramic brackets. Am J Orthod Dentofacial Orthop 2008;134:548-555.

11. Sorel O, El Alam R, Chagneau F, Cathelineau G. Comparison of bond strength between simple foil mesh and laser-structured base retention brackets. Am J Orthod Dentofacial Orthop 2002;122:260-266

12. Zachrisson BU, Buyukyilmaz T. Bonding in orthodontics. In:Graber TM, Vanarsdall RL, Vig KW, editors. Orthodontics: current principles and techniques. St Louis: Elsevier-Mosby; 2005;612-619.

13. Chen CS, Hsu ML, Chang KD, Kuang SH, Chen PT, Gung YW. Failure analysis: enamel fracture after debonding orthodontic brackets. Angle Orthod 2008;78:1071-1077.

14. Bishara SE, Ostby AW, Laffoon J, Warren JJ. Enamel cracks and ceramic brackets failure during debonding in vitro. Angle Orthod 2008;78:1078-1083.

15. Ahrari F, Heravi F, Fekrazad R, Farzanegan F, Nakhaei S. Does ultra-pulse $\mathrm{CO}(2)$ laser reduce the risk of enamel damage during debonding of ceramic brackets? Lasers Med Sci 2012;27:567-574.

16. Dumbryte I, Jonavicius T, Linkeviciene L, Linkevicius $\mathrm{T}$, Peciuliene V, Malinauskas M. The prognostic value of visually assessing enamel microcracks: do debonding and adhesive removal contribute to their increase? Angle Orthod 2016;86:437-447.

17. Silveira GS, Bittencourt LP, Mucha JN. Scoring of ceramic bracket bases for easier debonding. Journal of clinical orthodontics: JCO. 2014 Jul;48(7):441-442.

18. Maskeroni AJ, Meyrs CE, Lorton L. Ceramic bracket bonding: a comparison of bond strength with polyacrylic acid and phosphoric acid enamel conditioning. Am J Orthod Dentofacial Orthop 1990;97:168-175.

19. Viazis AD, Cavanaugh G, Bevis RR. Bond strength of ceramic bracket under shear stress: an in vitro report. Am J Orthod Dentofacial Orthop 1990;98:214-221.

20. Bordeau FM, Moore RN, Bagby MD. Comparative evaluation of ceramic bracket base designs. Am J Orthod Dentofacial Orthop 1994;105:552-560.

21. Wang WN, Meng CL, Torng TH. Bond strength: a comparison between chemical coated and mechanical interlock bases of ceramic and metal brackets. Am J Orthod Dentofacial Orthop 1997;111:374-381.

22. Guan G, Takano-Yamamoto T, Miyamoto M, Hattori T, Ishikawa K, SuzukiI K. Shear bond strengths of orthodontic plastic brackets. Am J Orthod Dentofacial Orthop 2000;117:438-443.

23. Swartz ML. Ceramic brackets. J Clin Orthod 1988;22:82-88.

24. Krell KV, Courey JM, Bishara SE. Orthodontic bracket removal using conventional and ultrasonic debonding techniques: enamel loss and time requirements. Am J Orthod Dentofac Orthop 1993;103:258-266.

25. Bishara S, Trulove T. Comparisons of different debonding techniques for ceramic brackets: an in vitro study, Part II. Am J Orthod Dentofacial Orthop. 1990;98:263-273

26. Sheridan JJ, Brawley G, Hastings J. Electrothermal debracketing: part I, an in vitro study. Am J Orthod Dentofac Orthop 1986;89:21-27. 